\title{
Time-Resolved Measurements Of Double Layer Evolution In Expanding Plasma
}

\author{
E. E. Scime
}

I. A. Biloiu

J. Carr

S. Chakraborty Thakur

M. Galante

See next page for additional authors

Follow this and additional works at: https://researchrepository.wvu.edu/faculty_publications

\section{Digital Commons Citation}

Scime, E. E.; Biloiu, I. A.; Carr, J.; Thakur, S. Chakraborty; Galante, M.; Hansen, A.; Houshmandyar, S.; Keesee, A. M.; McCarren, D.; and Sears, S., "Time-Resolved Measurements Of Double Layer Evolution In Expanding Plasma" (2010). Faculty Scholarship. 1033.

https://researchrepository.wvu.edu/faculty_publications/1033 
Authors

E. E. Scime, I. A. Biloiu, J. Carr, S. Chakraborty Thakur, M. Galante, A. Hansen, S. Houshmandyar, A. M. Keesee, D. McCarren, and S. Sears 


\title{
Time-resolved measurements of double layer evolution in expanding plasma ${ }^{a)}$
}

\author{
E. E. Scime, ${ }^{1, b)}$ I. A. Biloiu, ${ }^{1}$ J. Carr, Jr., ${ }^{1}$ S. Chakraborty Thakur, ${ }^{1}$ M. Galante, ${ }^{1}$ \\ A. Hansen, ${ }_{3}^{1}$ S. Houshmandyar, ${ }^{1}$ A. M. Keesee, ${ }^{1}$ D. McCarren, ${ }^{1}$ S. Sears, ${ }^{1}$ C. Biloiu, ${ }^{2}$ \\ and X. Sun ${ }^{3}$ \\ ${ }^{1}$ Department of Physics, West Virginia University, Morgantown, West Virginia 26506, USA \\ ${ }^{2}$ Varian Associates, Gloucester, Massachusetts 01930, USA \\ ${ }^{3}$ Tri-Alpha Corporation, Foothill Ranch, California 92610, USA
}

(Received 19 October 2009; accepted 30 November 2009; published online 6 January 2010)

\begin{abstract}
Observations in steady-state plasmas confirm predictions that formation of a current-free double layer in a plasma expanding into a chamber of larger diameter is accompanied by an increase in ionization upstream of the double layer. The upstream plasma density increases sharply at the same driving frequency at which a double layer appears. For driving frequencies at which no double layer appears, large electrostatic instabilities are observed. Time-resolved measurements in pulsed discharges indicate that the double layer initially forms for all driving frequencies. However, for particularly strong double layers, instabilities appear early in the discharge and the double layer collapses. () 2010 American Institute of Physics. [doi:10.1063/1.3276773]
\end{abstract}

\section{INTRODUCTION}

In its simplest form, a double layer (DL) consists of two spatially separated charge layers, one positive and one negative. Electrostatically, a DL acts very much like a sheath. However, whereas a conventional sheath appears at the surface of an object inserted into plasma or at plasma boundary, ${ }^{1}$ a DL is a freestanding structure and can appear anywhere within the plasma. Ions (electrons) created on the high (low) potential side of the DL are accelerated to the low (high) potential side. Ions (electrons) residing on the low (high) potential side are trapped by the potential barrier. These populations are typically referred to as the free and trapped species in the DL literature. A DL is just one mechanism whereby an accelerated population of ions can be created in a plasma. For example, in plasma expanding into a vacuum or along a divergent magnetic field, freely expanding electrons create an ambipolar electric field that accelerates ions out of the source at the sound speed, $C_{s}=\sqrt{k T_{e} / m_{i}}$. Therefore, a potential drop larger than $k T_{e} / e$ across a spatially localized region is often cited requirement for a plasma structure to be identified as a DL. Strong DLs can have potential drops much larger than the electron temperature, e.g., many hundreds of, if not thousands, times the electron thermal energy. ${ }^{2-5}$ Potential drops of less than $k T_{e}$ have also been reported for particular types of DLs, e.g., the slow ion acoustic type DL. ${ }^{6}$

Studies of DLs have been carried out in laboratory plasmas with densities ranging from $10^{6}$ to $10^{20} \mathrm{~cm}^{-3}$ and electron temperatures from several to hundreds of $\mathrm{eV}^{2,6,7} \mathrm{Ob}-$ served DL thickness have ranged from several ${ }^{8}$ to thousands of Debye lengths. ${ }^{3}$ In laboratory experiments and simulations, DLs are typically produced in systems driven by electric current, externally imposed potential differences, or by

\footnotetext{
a) Paper TI2 3, Bull. Am. Phys. Soc. 54, 253 (2009).

${ }^{b)}$ Invited speaker.
}

externally imposed electron temperature differences (due to multiple plasma sources). Some of the first measurements of DL potential structure were performed in a double plasma source. The relatively low plasma density of the double plasma source yielded a wide DL structure that was well suited for investigation with in situ probes. ${ }^{9}$ Target plasmas for later investigations of DLs improved greatly with the introduction of the triple plasma (TP) device. The type of DL created in the TP was controllable by varying the potential on the biasable grids. Strong $\left(e \phi / T_{e}>10\right),{ }^{10}$ weak $\left(e \phi / T_{e} \sim 5\right),{ }^{11}$ one-dimensional to two-dimensional, ${ }^{12}$ and stationary to moving DLs (Ref. 13) were produced in TP devices. DLs were also observed in freely expanding laboratory plasmas if the plasma source contained both Maxwellian and energetic electrons. Hairapetian and Stenzel ${ }^{14}$ studied the temporal and spatial evolution of a DL as a pulsed plasma expanded into a chamber with a low background neutral pressure $\left(10^{-6} \mathrm{~cm}^{-3}\right)$. Time-resolved measurements of the DL potential profile revealed that the DL propagated into the chamber at approximately the ion sound speed and reached steady state after $200 \mu$ s. The DL formed along U-shape equipotential contours aligned with the expansion direction. Hairapetian and Stenzel ${ }^{14}$ concluded that the DL arose along the expansion front of the plasma where thermal electrons were trapped by a large potential drop due to the energetic tail electrons. They suggested that the DL was generated self-consistently to maintain the separation of the distinct electron populations.

Solutions to the Poisson equation that yield a onedimensional DL in a collisionless plasma can obtained from fluid theory ${ }^{15}$ or through a Bernstein-Greene-Kruskal (BGK) analysis. ${ }^{16}$ A key feature of the BGK DL solution is that at least three of the four particle populations, i.e., the free and trapped ions and electrons, must be present. The free particles, i.e., beams accelerated by passage through the DL, 

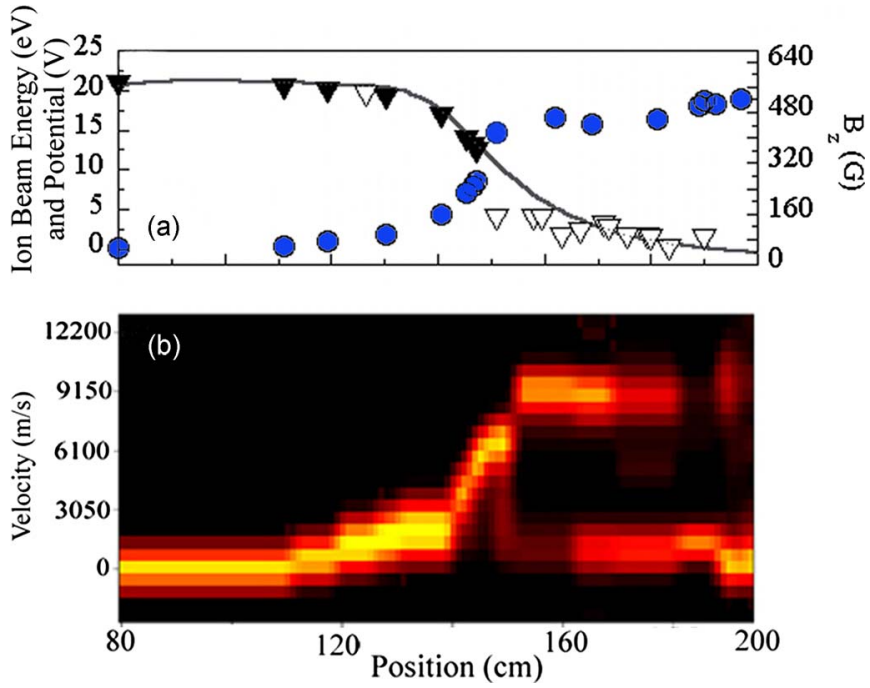

FIG. 1. (Color online) (a) Plasma potential vs axial position in an expanding plasma as measured with a rf-compensated, planar Langmuir probe (open triangles), ion beam energy as measured with LIF (filled circles), predicted upstream plasma potential based on ion beam data (solid triangles), and axial magnetic field strength (solid line). (b) Natural logarithm of amplitude of parallel ion velocity distribution function vs parallel velocity and axial position as measured by LIF. Figure adapted from Ref. 19 .

are routinely used as an experimental signature of the presence of a DL.

Through retarding field energy analyzer (RFEA) ${ }^{17,18}$ and nonperturbative laser induced fluorescence (LIF) (Ref. 19) measurements of ion energy and velocity distribution functions (IVDFs), recent laboratory experiments have demonstrated that stationary, current-free DLs form spontaneously in helicon plasmas expanding along a diverging magnetic field when the source is operated at pressures below a few millitorr. These are single source, pulsed or steady-state, plasma experiments without any applied dc potentials and the only significant imposed inhomogeneity in the devices is the divergent magnetic field. A LIF measurement of the structure of a spontaneous DL in an expanding helicon plasma structure is shown in Fig. 1. Clearly evident are the stationary upstream ion population (left side of the figure), the downstream trapped population (stationary population on right side of the figure), and the ions accelerated in the presheath and sheath regions of the DL (the ion beam). For one group of Zeeman split states of the ion line probed by LIF, the acceleration of the ions in the weakening magnetic field results in a balance between oppositely directed Zeeman and Doppler shifts of the LIF absorption line. The magnitude of the asymmetric optical pumping that arises from this effect (also employed in magneto-optical traps) provides an absolute measurement of the ion collisionality in the DL region. The collision frequencies measured in this fashion confirm that the ion mean free path is comparable to the density gradient scale length when the DL appears at low neutral pressures. ${ }^{20}$ The beamlike nature of the accelerated ion population is evident in the tomographic IVDF measurement shown in Fig. 2. The beam and the background ion populations are isotropic in velocity space and are distinct, independent populations.

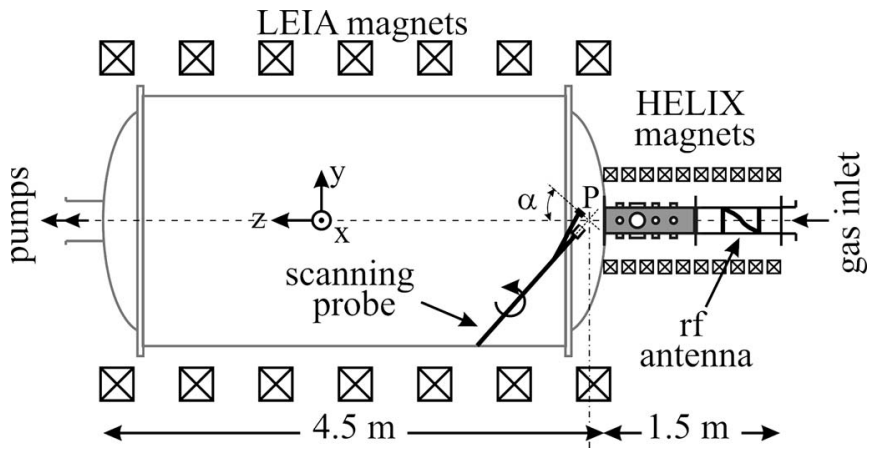

FIG. 2. Tomographic measurement of the two-dimensional IVDF obtained $19 \mathrm{~cm}$ downstream of the DL in an expanding helicon plasma. The negative value for the fast population axial velocity arises from ion flow out of the source toward the direction from which the laser is injected (the $\alpha$ direction). Reprinted with permission from Bilious et al., Plasma Sources Sci. Technol. 18, 025012 (2009). Copyright @ 2009 Institute of Physics.

The spontaneous formation of these DLs is observed for a wide range of ion species; ${ }^{21}$ in multiple helicon sources in different laboratories; ${ }^{22,23}$ in combination with physical apertures that create additional accelerating sheathlike structures $;{ }^{24}$ and through the introduction of strongly electronegative molecules to create a steep and localized electron density gradient. ${ }^{25}$ Recent experiments demonstrated that the DL extends across a wide swath of the divergent field lines and that the resultant U-shaped potential structure is consistent with Fast Auroral SnapshoT satellite measurements of naturally occurring DL structures in the auroral zone. ${ }^{26}$

However, certain aspects of the observed ion acceleration process are inconsistent with expectations for a "classic" DL, e.g., in the LIF measurements the ion acceleration extends over many tens to hundreds of Debye lengths ${ }^{19}$ instead of the $10-50$ D lengths ${ }^{15}$ expected in a classic DL. In addition, although the mean free paths of the plasma constituents has been shown to play a critical role in triggering the formation of the DL (through the experimental observations of a critical neutral pressure for DL formation), ${ }^{19,27-30}$ only recently has a first-principles theoretical explanation for DL formation been proposed.

Lieberman and Charles $^{31}$ developed a diffusioncontrolled model that describes the formation of a currentfree DL in a plasma expanding from a small-diameter, dielectric source chamber to a large-diameter, conducting expansion chamber; similar to our experimental configuration and the configuration of plasma sources used in the plasma processing industry and in other expanding helicon plasma laboratory experiments. The diffusion-controlled model couples the dynamics of the particles in the nonneutral DL to the diffusive flows of the quasineutral plasma in the source and expansion chambers. As in a conventional DL model, the DL is embedded in a quasineutral plasma consisting of four groups of charged particles: thermal ions, monoenergetic accelerated ions flowing downstream, accelerated electrons flowing upstream, and thermal electrons. To ensure that the DL is current-free, the model adds another group of counterstreaming electrons formed by the reflection of almost all of the accelerated electrons from the sheath at the insulated end wall of the source chamber. The potential 
difference across the DL is determined by the upstream and downstream particle balances. Since the upstream radius is smaller than the downstream radius, ionization by thermal electrons upstream is not enough to balance the larger particle losses upstream. An additional source of upstream ionization becomes necessary and is provided by the accelerated group of electrons. In the model, the DL vanishes at very low pressures as the maximum ionization rate for the accelerated electrons upstream is insufficient to balance the excess upstream particle loss. At very high pressures, the system length becomes comparable to the energy relaxation length for ionizing electrons. Since electrons are heated upstream, the downstream ionizing electron density decreases at higher pressures. When the downstream and upstream ionization rates become equal to the corresponding particle loss rates, the additional ionization provided by the accelerated electrons is unnecessary and the DL vanishes.

In this work, we first present our observations of increased upstream (on the high potential side of the DL) ionization only when an ion beam is observed downstream, i.e., when a DL is present, in steady-state discharges. ${ }^{32}$ For identical magnetic field geometries, neutral pressures, and $\mathrm{rf}$ powers, the DL is turned on and off by varying the antenna frequency in the helicon source. Coincident with the DL vanishing, large, ion-acoustic-like, electrostatic instabilities are observed. We then present time-resolved, observations in pulsed helicon source plasmas that indicate that the DL forms for all antenna frequencies, but for intense and energetic ion beams the DL collapses as a large, coherent, electrostatic instability appears. Lower energy and relatively less intense ion beams, created at higher antenna frequencies, persist throughout the discharge and no instabilities are observed. These observations are consistent with excitation of a current-driven instability that appears at large beam current densities and disrupts the DL.

\section{EXPERIMENTAL APPARATUS}

These experiments were performed in the Hot hELIcon eXperiment (HELIX). HELIX consists of a $61 \mathrm{~cm}$ long, Pyrex vacuum chamber $10 \mathrm{~cm}$ in diameter and connected to a $91 \mathrm{~cm}$ long, $15 \mathrm{~cm}$ diameter stainless steel chamber. The stainless steel chamber opens into a $2 \mathrm{~m}$ diameter, $4.5 \mathrm{~m}$ long expansion chamber (Fig. 3). Ten electromagnets produce a steady state axial magnetic field of $0-1300 \mathrm{G}$ in the source. Plasmas are created at neutral pressures (with $\mathrm{rf}$ on) ranging from 0.1 to 100 mTorr. rf power of up to $2.0 \mathrm{~kW}$ over a frequency range of $6-18 \mathrm{MHz}$ is coupled into a $19 \mathrm{~cm}$ half wave, helical antenna to create the steady state plasma. Characteristic electron temperature and densities in the source are $T_{e} \approx 5 \mathrm{eV}$ and $n \approx 1 \times 10^{13} \mathrm{~cm}^{3}$ as measured with rf compensated Langmuir probes located $50 \mathrm{~cm}$ downstream of the antenna, but upstream of the expansion region.

A typical LIF IVDF measurement (see Ref. 33 for a complete description of the LIF system) consists of sweeping the frequency of a very narrow bandwidth laser through a collection of ions or atoms that have a thermally broadened velocity distribution function. The illuminated ions or atoms absorb a photon and are pumped into an excited state when

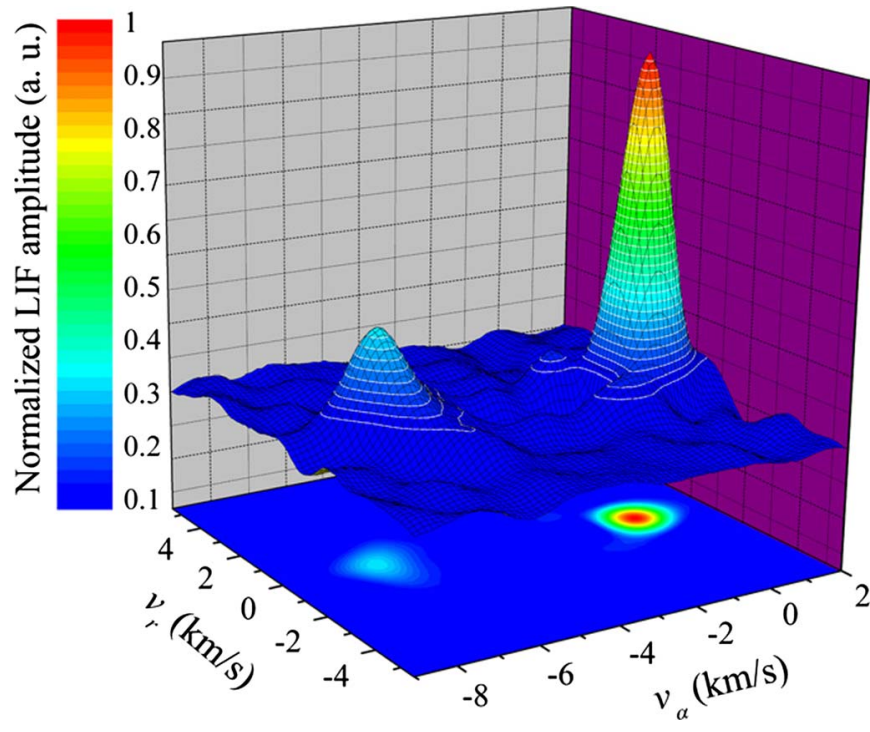

FIG. 3. (Color online) Combined HELIX-LEIA system with the location of the downstream $\left(\mathrm{P}_{1}\right)$ and upstream $\left(\mathrm{P}_{2}\right)$ LIF measurement locations indicated.

the laser appears at the appropriate frequency in their respective rest frames. $90 \%$ of the laser light is mechanically chopped for lock-in amplification and injected along the axis of the experiment. The fluorescent emission from the pumped excited state is measured with a filtered photomultiplier tube that is fiber optically coupled to a variety of in situ probes and optical assemblies distributed along the axis of the experiment. ${ }^{34}$ The remaining $10 \%$ of the beam is passed through an iodine cell for a consistent zero-velocity reference ${ }^{35}$ For steady-state plasmas, the laser is modulated at $\sim 2 \mathrm{kHz}$. For time-resolved LIF in pulsed discharges, the laser is modulated at $40 \mathrm{kHz}$ and the time resolution of the measurements is limited to a few milliseconds by the $1 \mathrm{~ms}$ integration time of the lock-in amplifier (needed to obtain sufficient photon counting statistics). ${ }^{36,37}$ The LIF emission as a function of laser frequency is fit to a either a single or pair of drifting Maxwellian distributions

$$
I_{R}(\nu)=I\left(\nu_{o}\right) e^{-m c^{2}\left(\nu-\nu_{o}\right)^{2} / 2 k_{B} T_{i} \nu_{o}^{2}},
$$

where $\nu_{o}$ is the rest frame frequency of the absorption line, $m_{i}$ the ion mass, and $T_{i}$ the ion temperature. For the magnetic field strengths of less than $100 \mathrm{G}$ in the expansion region, Zeeman splitting of the two circularly polarized absorption lines is ignorable for the ion temperature determination. In the plasma source, the measured line shifts must be corrected for the Zeeman shift of the absorption line.

Independent measurements of the IVDF are obtained with a RFEA downstream of the ion acceleration region and aligned so that the RFEA aperture faces the upstream (helicon source) direction. The RFEA consists of four closely spaced mesh screens (250 lines per inch nickel mesh glued to copper support rings) placed behind a grounded aperture and held in grounded probe assembly. ${ }^{17,18}$ The RFEA probe and associated electronics are described in detail in Ref. 18.

The downstream electron temperature and densities are measured with a rf-compensated Langmuir probe at multiple 


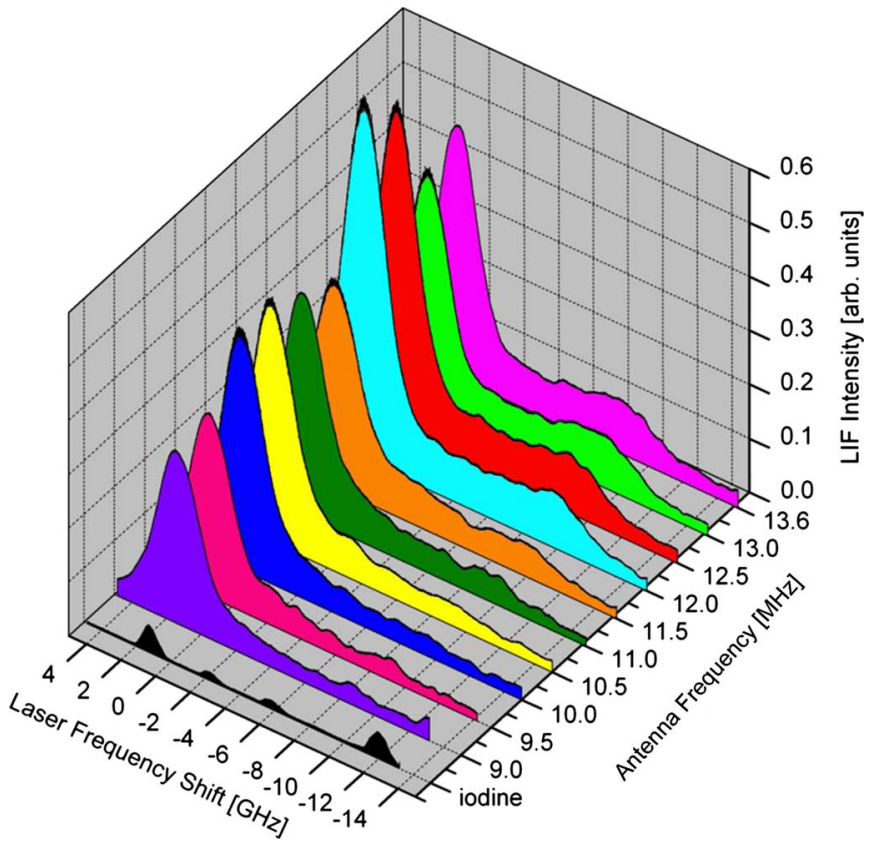

FIG. 4. (Color online) LIF measurements of the downstream IVDF vs antenna frequency obtained $124 \mathrm{~cm}$ downstream of the antenna. The laser frequency shift in gigahertz is relative to the rest frame frequency of the LIF absorption line. The reference iodine spectrum is also shown. The distinct fast ion population, the ion beam, is observed only for antenna frequencies above $\sim 11 \mathrm{MHz}$. Reprinted with permission from Chakraborty Thakur et al., Phys. Rev. Lett. 102, 035004 (2009). Copyright (C) 2009 American Physical Society.

locations along the axis of the expansion chamber. ${ }^{38}$ The power spectrum of the electric field fluctuations is measured with a multitip probe in the plasma source; $50 \mathrm{~cm}$ downstream of the rf antenna.

\section{FREQUENCY THRESHOLD FOR DOUBLE LAYER FORMATION}

Investigations into the relationship between the antenna frequency and DL formation were carried out in argon plasma with a nominal rf power of $750 \mathrm{~W}$, a source magnetic field of $700 \mathrm{G}$, and a magnetic field of $50 \mathrm{G}$ in the expansion region. With a constant flow rate of 3.6 (SCCM) (SCCM denotes standard cubic centimeters per minute at STP) into the system, the neutral pressures in the source and expansion regions were 0.2 and 0.05 mTorr, respectively. For just the upstream LIF-based IVDF measurements (to improve the LIF signal-to-noise), the magnetic field in the expansion region was decreased to $14 \mathrm{G}$, the rf power lowered to $700 \mathrm{~W}$, and the flow rate increased to 8.6 SCCM; yielding neutral pressures in the source and expansion regions of 1.0 and 0.09 mTorr, respectively. During the scans of the antenna frequency, all other source parameters were held constant.

LIF measurements of the Doppler-shifted (relative to the rest frame absorption line frequency), downstream IVDF are shown in Fig. 4. Because the laser injection direction is opposite to the flow of the plasma, a negative frequency shift corresponds to ion flow toward the expansion chamber. The

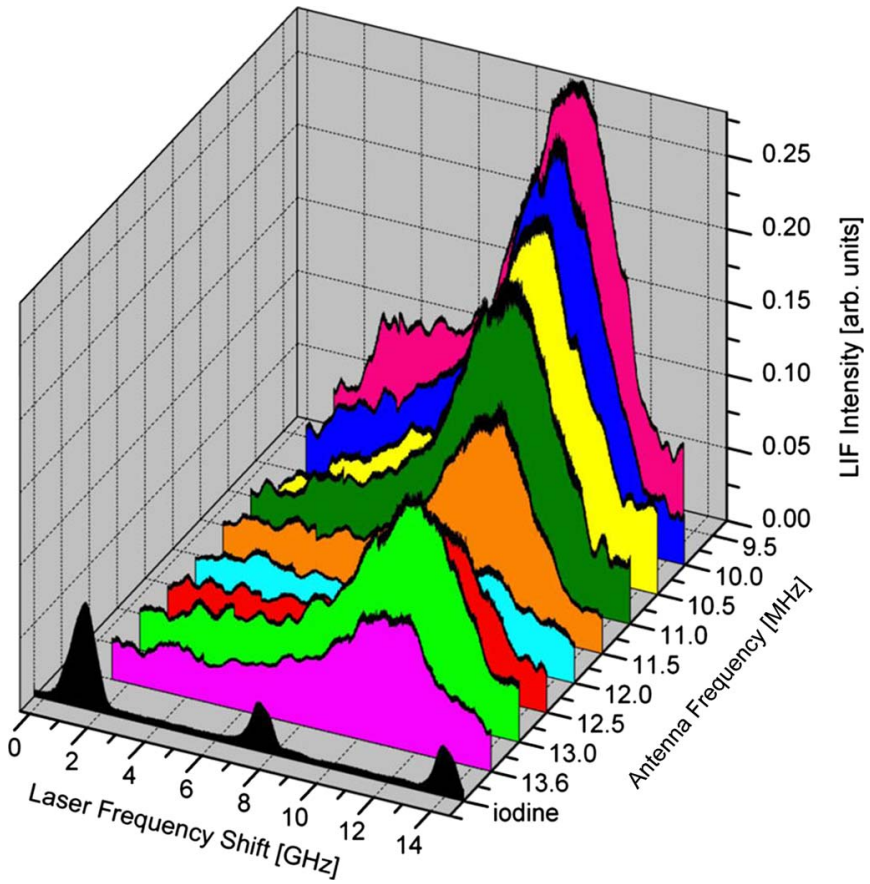

FIG. 5. (Color online) LIF measurements of the upstream IVDF vs antenna frequency obtained $95 \mathrm{~cm}$ downstream of the antenna. The reference iodine spectrum is also shown. A broad tail extending to very small velocities appears only for the lower antenna frequencies. Reprinted with permission from Chakraborty Thakur et al., Phys. Rev. Lett. 102, 035004 (2009). Copyright (C) 2009 American Physical Society.

large amplitude peaks at low velocities (small frequency shift) are the background ion population. For antenna frequencies below $11.5 \mathrm{MHz}$, there is a strong background population and a long tail of energetic ions. For antenna frequencies of $12 \mathrm{MHz}$ and higher, a distinct ion beam appears at large, downstream directed, velocities. Based on fits to a pair of drifting Maxwellian distributions and correcting the measured bulk flow speeds for the angle $\alpha$ of the scanning probe $\left[V_{\text {actual }}=V_{\text {obs }} / \cos (\alpha) ; \alpha=47^{\circ}\right]$ (probe location is shown in Fig. 3), the ion beam velocity increases from approximately $7.7 \mathrm{~km} / \mathrm{s}$ at $13.56 \mathrm{MHz}$ to $9.0 \mathrm{~km} / \mathrm{s}$ at $12 \mathrm{MHz}$. Since previous measurements demonstrated that the beam velocity increases for weaker expansion chamber magnetic fields, ${ }^{39}$ the downstream beam velocities would have been even larger had the expansion chamber field been reduced to the $14 \mathrm{G}$ expansion chamber magnetic field strengths used for the upstream IVDF measurements described below.

Shown in Fig. 5 are the upstream LIF measurements of the IVDF. A positive shift in the frequency of the peak LIF emission corresponds to ion flow toward the expansion chamber. Note that the antenna frequency axis has been reversed compared with Fig. 4 so that the entire IVDF can be seen for each measurement. A single, high-speed ion population dominates the measured IVDF. Comparison to the DL structure shown in Fig. 1 places these measurements within the acceleration region just upstream of the DL. After correcting for the Zeeman shift of the absorption line for a magnetic field of $700 \mathrm{G}$, there is a slight increase in the 


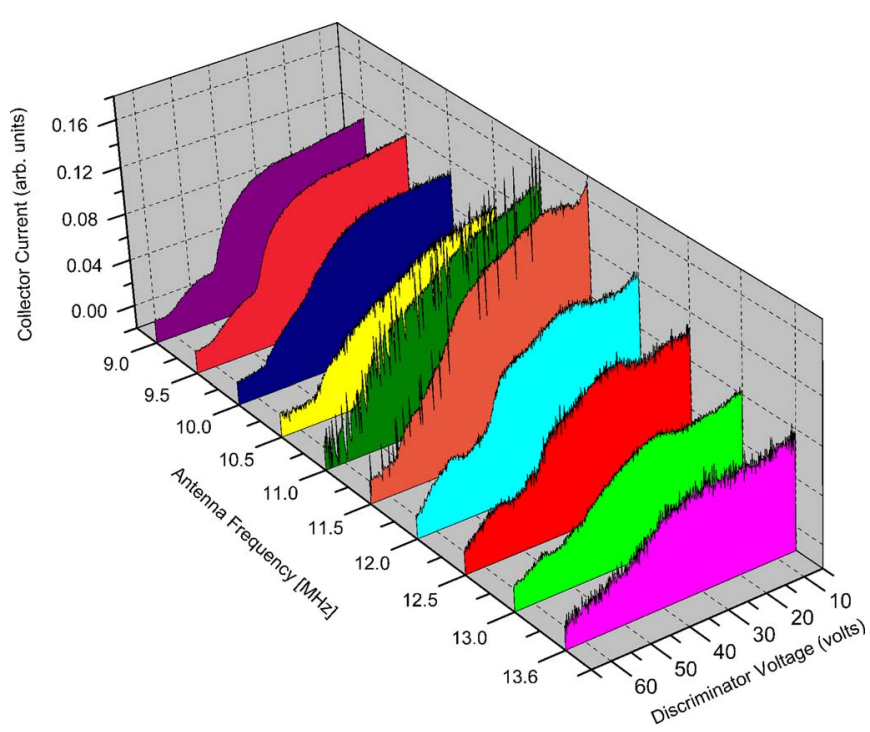

FIG. 6. (Color online) RFEA collector current vs discriminator voltage as a function of the antenna frequency obtained $184 \mathrm{~cm}$ downstream of the antenna. The onset of large scale electrostatic fluctuations occurs at $11.5 \mathrm{MHz}$.

downstream-directed drift velocity of the bulk ion population with increasing antenna frequency from $6.7 \mathrm{~km} / \mathrm{s}$ at $9.5 \mathrm{MHz}$ to $7.5 \mathrm{~km} / \mathrm{s}$ at $12 \mathrm{MHz}$. The bulk drift velocity remains nearly constant for further increases in the antenna frequency. The increase in LIF signal amplitude at lower antenna frequencies likely results from an increase in the population of the initial metastable argon ion state of the LIF sequence (since lower antenna frequencies typically result in improved coupling of the rf power into the plasma ${ }^{40}$ ) or the slightly longer time spent in front of the collection optics by slower moving excited state ions. Note that for antenna frequencies below $11.5 \mathrm{MHz}$, a broad tail of slower ions appears. By $9.5 \mathrm{MHz}$, the tail of slow ions expands to include ions moving in the upstream direction as well. The increase in the drift velocity with increasing antenna frequency is opposite to what was observed in case of the ion beam from the downstream LIF measurements.

Shown in Fig. 6 are RFEA collector current measurements versus discriminator voltage for the same range of antenna frequencies. The data shown were averaged over 100 measurements at each antenna frequency. There is a clear onset of large amplitude fluctuations in the RFEA collector current at driving frequencies of 11.0-11.5 MHz. This $\sim 11.5 \mathrm{MHz}$ threshold corresponds to the same threshold frequency above which two distinct ion populations are evident in the RFEA data: one is the background ion population that appears in the RFEA data at the plasma potential of approximately $25 \mathrm{~V}$ and the other is an ion beam population that appears at larger discriminator voltages. At lower antenna frequencies, only the background ion population appears in the RFEA data. The parent ion velocity distribution functions obtained from fits of the full theoretical expression for RFEA collector current versus discriminator voltage for a stationary plasma with a fast ion population ${ }^{18}$

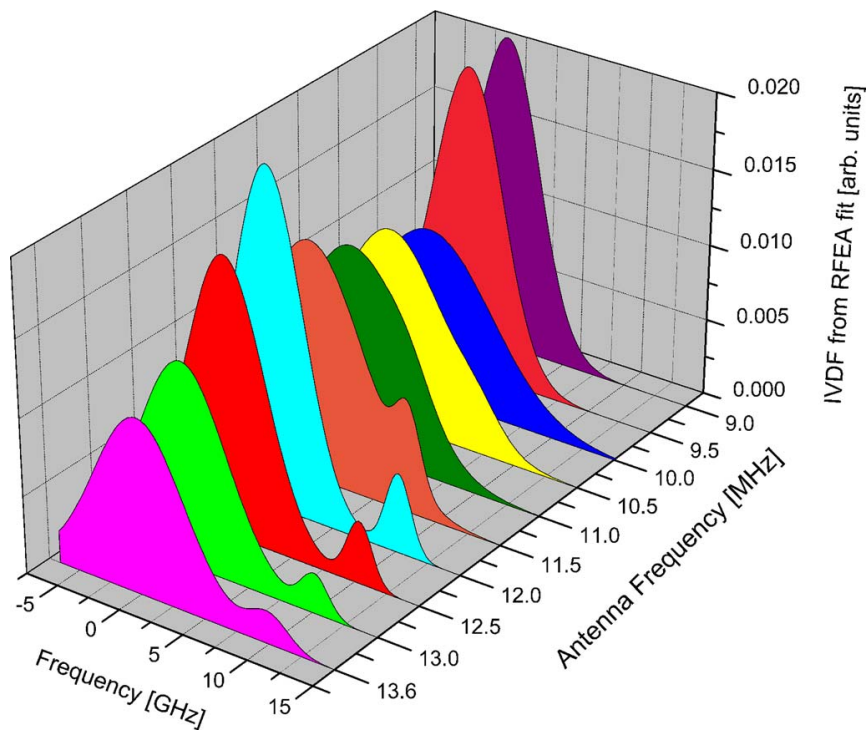

FIG. 7. (Color online) RFEA measurements [the bi-Maxwellian which gives the best fit of Eq. (2) to the raw data in Fig. 6] of the downstream IVDF vs antenna frequency obtained $184 \mathrm{~cm}$ downstream of the antenna. The ion beam appears only for the higher antenna frequencies.

$$
\begin{aligned}
I\left(\Phi_{D}\right)= & \sqrt{\frac{e^{2}}{2 m \pi}}\left[( \frac { n _ { p } } { 2 } ) \left\{\sqrt{T_{P}} e^{-\left[\left(\sqrt{e \Phi_{D}-e \Phi_{P}}-\sqrt{E_{P}}\right)^{2} / T_{P}\right]}\right.\right. \\
& \left.+\sqrt{E_{P} \pi} \operatorname{erfc}\left[\left(\sqrt{e \Phi_{D}-e \Phi_{P}}-\sqrt{E_{P}}\right) / \sqrt{T_{P}}\right]\right\} \\
& +n_{b}\left\{\sqrt{T_{b}} e^{-\left[\left(\sqrt{e \Phi_{D}-e \Phi_{P}}-\sqrt{E_{b}}\right)^{2} / T_{b}\right]}\right. \\
& \left.\left.+\sqrt{E_{b} \pi} \operatorname{erfc}\left[\left(\sqrt{e \Phi_{D}-e \Phi_{P}}-\sqrt{E_{b}}\right) / \sqrt{T_{b}}\right]\right\}\right],
\end{aligned}
$$

are shown in Fig. 7. The RFEA-derived IVDFs are consistent with the downstream LIF IVDF measurements. For antenna frequencies below $11.5 \mathrm{MHz}$, there is a single, low velocity, background ion population. For antenna frequencies above $12 \mathrm{MHz}$, a distinct ion beam appears at large, downstreamdirected velocities. From the fits to the RFEA measurements, the ion beam velocity increases from approximately $5.5 \mathrm{~km} / \mathrm{s}$ at $13.56 \mathrm{MHz}$ to $6.4 \mathrm{~km} / \mathrm{s}$ at $12 \mathrm{MHz}$. The roughly $1 \mathrm{~km} / \mathrm{s}$ increase in the ion beam velocity matches the trend seen in the downstream LIF measurements.

The upstream and downstream plasma densities are shown in Fig. 8. There is gradual increase in the downstream plasma density with increasing antenna frequency from $6 \times 10^{9} \mathrm{~cm}^{-3}$ at $9 \mathrm{MHz}$ to $1 \times 10^{10} \mathrm{~cm}^{-3}$ at $13.56 \mathrm{MHz}$. Upstream of the expansion region; there is a large and discontinuous increase in the measured density as the antenna frequency changes from 11.5 to $12 \mathrm{MHz}$. This is the same driving frequency at which the downstream ion beam, and by implication the DL, appears. With the DL present, the density increased to $2.6 \times 10^{11} \mathrm{~cm}^{-3}$ from an average density of $0.85 \times 10^{11} \mathrm{~cm}^{-3}$ without the DL; an increase of nearly a factor of 3. It is important to remember that this increase in plasma density occurs without any change in the magnetic field geometry, neutral pressure, or forward rf power.

The upstream and downstream electron temperatures, measured with the same Langmuir probes, also show a dis- 


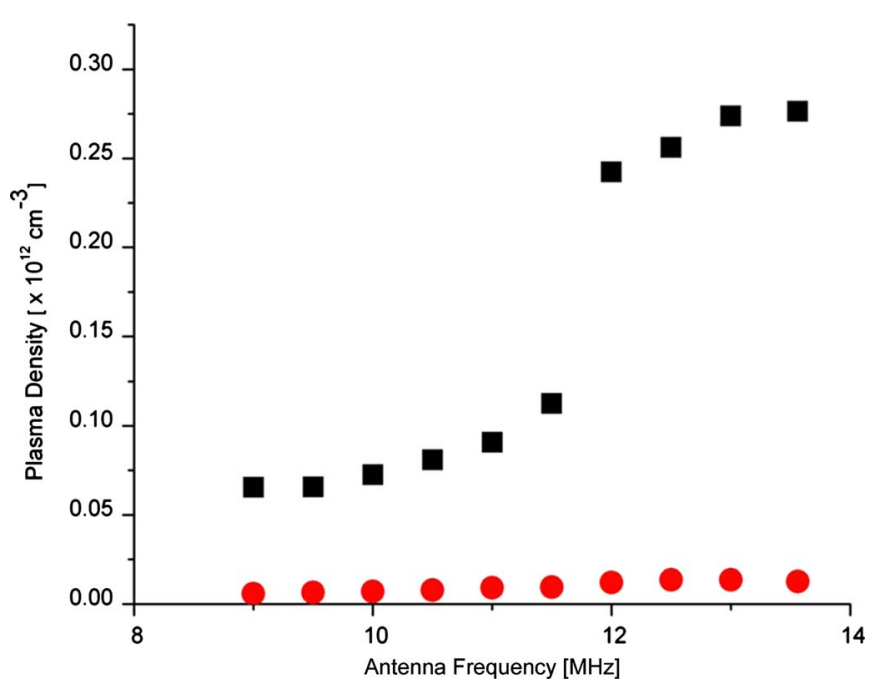

FIG. 8. (Color online) Upstream (squares) and downstream (circles) plasma density vs antenna frequency for rf power of $750 \mathrm{~W}, B_{\mathrm{HELX}}=700 \mathrm{G}$, $B_{\text {LEIA }}=50 \mathrm{G}$, and a pressure of 0.2 mTorr. The error bars for each measurement are smaller than the size of the data points shown. Reprinted with permission from Chakraborty Thakur et al., Phys. Rev. Lett. 102, 035004 (2009). Copyright (C) 2009 American Physical Society.

tinct change in the upstream electron temperature at driving frequencies above $12 \mathrm{MHz}$. The electron temperature drops from approximately $10.5 \mathrm{eV}$ when the DL is not present to an average of $8.7 \mathrm{eV}$ for driving frequencies at which the ion beam is observed downstream. The downstream electron temperature is relatively constant at an average value of 7.9 $\mathrm{eV}$ for all driving frequencies.

The IVDF and upstream density measurements are consistent with the diffusion-controlled model prediction of enhanced upstream plasma density arising from the formation of a current-free DL in expanding plasma. ${ }^{31,32}$ For identical plasma source parameters, apart from the driving frequency, the upstream density clearly increases when the downstream LIF and RFEA measurements indicate the presence of a current-free DL.

\section{INSTABILITIES ASSOCIATED WITH DOUBLE LAYER FORMATION}

During these experiments, it was noticed that the upstream and downstream Langmuir probe measurements were extraordinarily noisy for source frequencies up to $11.5 \mathrm{MHz}$. Each measurement had to be repeated many times to obtain reliable average density and electron temperature values. Above source frequencies of $12 \mathrm{MHz}$, a single measurement was sufficient. Shown in Fig. 9 are the multitip probe measurements of the power spectra of the upstream electric field fluctuations versus driving frequency. For driving frequencies below $11.5 \mathrm{MHz}$, the spectra are dominated by a wave at a fundamental frequency of $17.5 \mathrm{kHz}$ and its harmonics. Coincident with the appearance of the current-free DL, there is a dramatic reduction in the amplitude of the floating potential fluctuations both upstream and downstream of the expansion region The $17.5 \mathrm{kHz}$ wave propagates primarily in the axial direction with a wavelength of approximately $3 \mathrm{~cm}$.

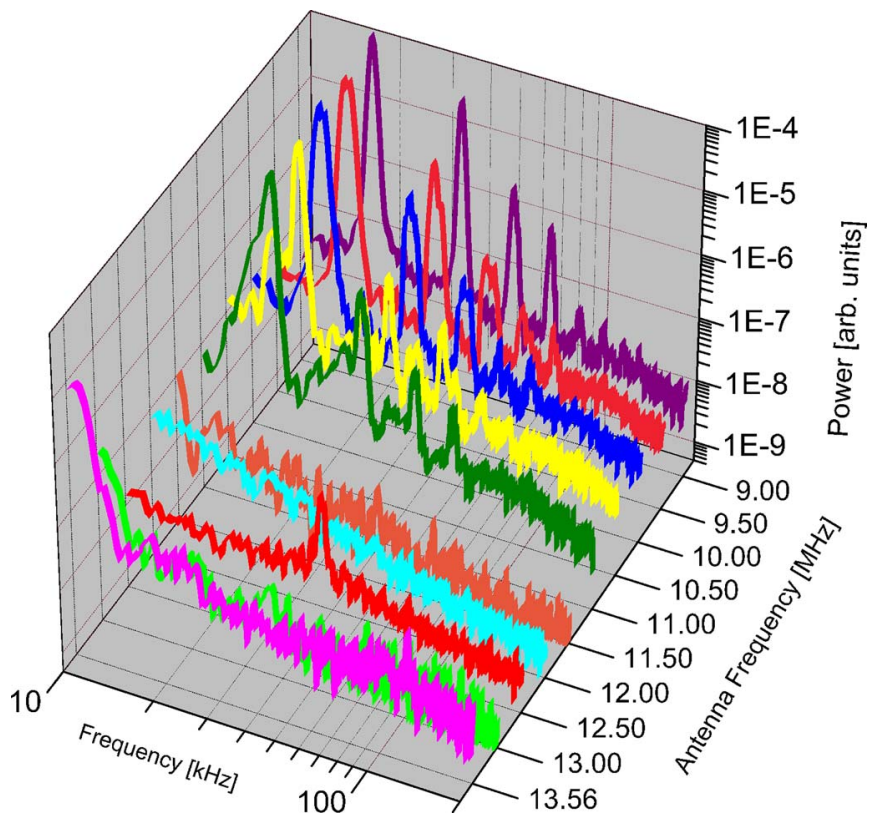

FIG. 9. (Color online) The power spectrum of the electrostatic instabilities vs antenna frequency obtained $50 \mathrm{~cm}$ downstream of the antenna. The instability at $17.5 \mathrm{kHz}$ and its harmonics appears only for the lower antenna frequencies.

We note that if the Bohm criterion (ions entering a sheath with speeds larger than the ion sound speed) is satisfied for a DL, it is possible that current driven instabilities will be excited in the DL, e.g., ion acoustic and cyclotron instabilities. Since there are four different populations of particles, excitation of a two-stream instability is also possible. Among these instabilities, the threshold for the Buneman instability yields the same current criterion as the Bohm criterion. Other instabilities, e.g., the Pierce instability, may trap ions in a narrow strongly varying potential and form a laminar DL. ${ }^{41}$ Once formed, the charge separation of the DL can persist without the presence of the Pierce instability since the charge distribution of a DL is one of the solutions of the BGK equation. Ion-acoustic driven DLs, characterized by an ion hole on the low potential side and a potential difference equal to or less than the electron temperature, ${ }^{42}$ are also observed to coincide with the existence of ion acoustic instabilities in computer simulations. ${ }^{43}$ Experimentally, ion cyclotron instabilities have been observed in TP device DL experiments in an inhomogeneous magnetic field. ${ }^{44}$

For these waves, their axial propagation rules out drift waves as the source of the instabilities. The short parallel wavelength and multiharmonic excitation are inconsistent with the characteristics of the ionization instability observed in other DL experiments. ${ }^{45}$ The multiple harmonics spanning frequencies below and above the ion cyclotron frequency are inconsistent with electrostatic ion cyclotron waves. Given that the wavelengths are much larger than the Debye length but much shorter than the system size, the fact that they propagate along the background magnetic field, and that excitation of the waves appears to be associated with a threshold particle drift velocity (ion beam speed) comparable to the ion sound speed, the waves are most likely due to a beam driven ion acoustic instability. ${ }^{46}$ 


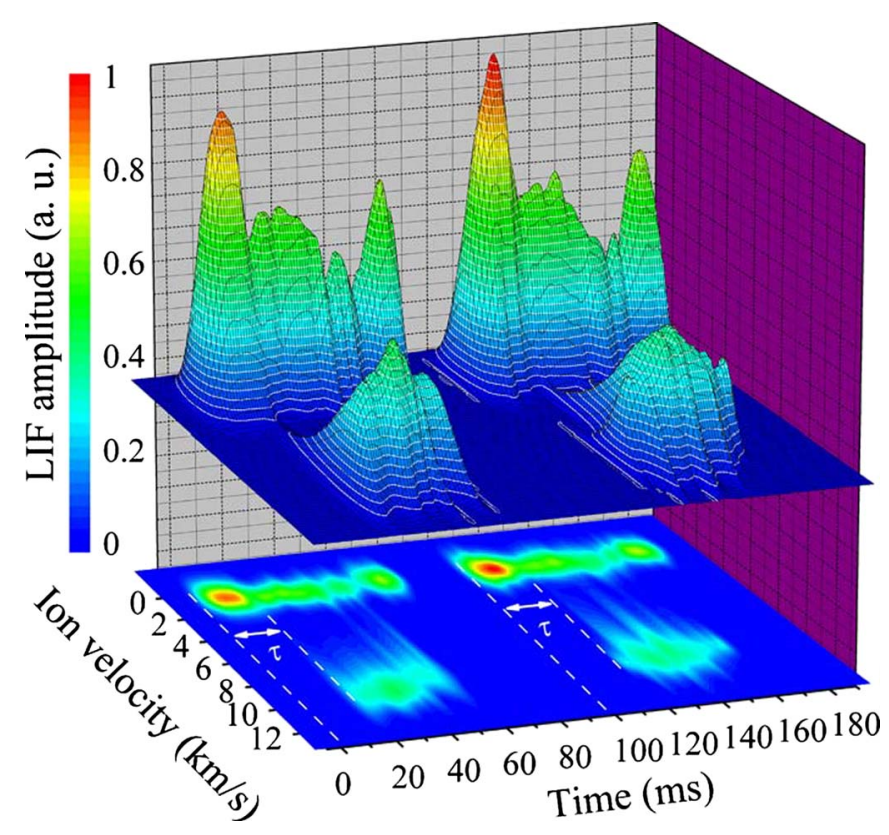

FIG. 10. (Color online) The evolution of the downstream parallel IVDF during two cycles of a $50 \mathrm{~ms}$ plasma pulse: top-surface plot showing fast $(\sim 10 \mathrm{~km} / \mathrm{s})$ and slow $(\sim 0.5 \mathrm{~km} / \mathrm{s})$ ion populations; bottom-a contour projection showing the time lag $(\tau \sim 19 \mathrm{~ms})$ in the appearance of the fast ion population. Figure adapted from Ref. 37.

The dispersion relation for the ion acoustic mode, for ion temperatures much smaller than the electron temperature, is

$$
\omega^{2}=\frac{1}{\left(1+\gamma \lambda_{D e}^{2} k^{2}\right)}\left(\frac{\gamma \kappa_{B} T_{e}}{m_{i}}\right) k^{2},
$$

where $\omega$ is the wave frequency, $k$ is the wave number, $\lambda_{D e}$ is the electron Debye length, $\gamma$ is the ratio of the specific heats, $\kappa_{B}$ is the Boltzmann constant, and $T_{e}$ is the electron temperature.

For large values of $k$, the frequency asymptotically approaches the ion plasma frequency. For frequencies much less than the ion plasma frequency $(\sim 60 \mathrm{MHz}$, in this experiment) and for values of $k$ smaller than $1 / \lambda_{D e}$, ( $\sim 12 \mathrm{~cm}^{-1}$ for these experimental conditions) the wave frequency is linear in $k$ and the phase velocity is approximately given by the ion sound speed

$$
\frac{\omega}{k}= \pm \sqrt{\frac{\gamma \kappa_{B} T_{e}}{m_{i}}} .
$$

For these experimental conditions, the ion sound speed is $6.4 \mathrm{~km} / \mathrm{s}$. The measured average phase velocity of the $17.5 \mathrm{kHz}$ wave is $7 \pm 1 \mathrm{~km} / \mathrm{s}$, which strongly suggests that these waves are ion acoustic waves.

\section{TIME-RESOLVED DOUBLE LAYER OBSERVATIONS}

In previous experiments, we demonstrated that formation of the DL in pulsed helicon source plasmas occurs a few ms after the initiation of the discharge (see Fig. 10 for an example of a time-resolved IVDF measurement of a beam and background ion population). To determine if there is a causal relationship between the DL and the observed ion acoustic waves, the complete IVDF and the plasma potential
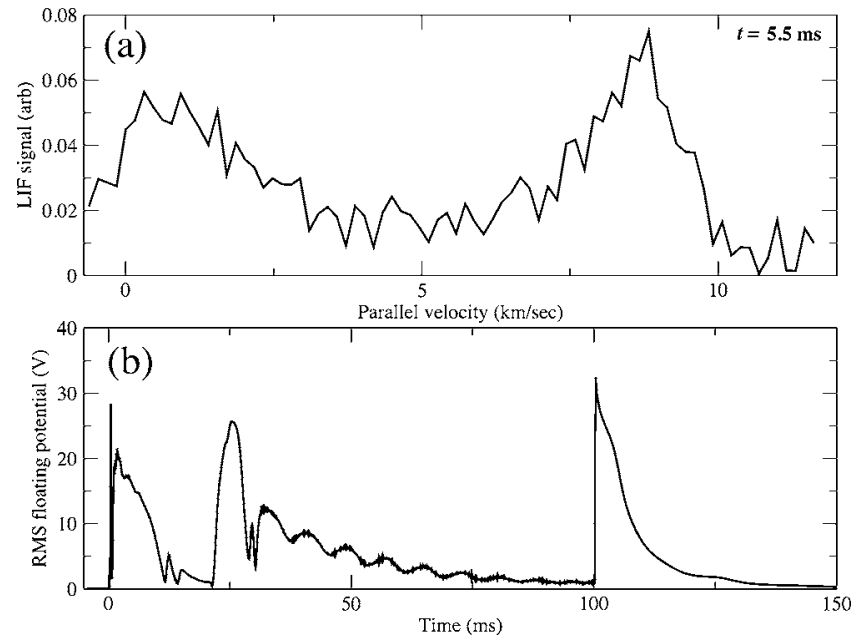

FIG. 11. For an antenna frequency of 9.0 MHz. (a) The downstream IVDF $5.5 \mathrm{~ms}$ after the start of the $100 \mathrm{~ms}$ long pulsed discharge. Both the background population (left) and ion beam (right) are evident. (b) Time series of the fluctuating floating potential from a single tip of the multitip probe averaged over 200 pulses. The large spike in fluctuation amplitude begins at $21.7 \mathrm{~ms}$ into the discharge. The large spike at $t=100 \mathrm{~ms}$ is the termination of the pulse.

fluctuation amplitude were measured as a function of time throughout $100 \mathrm{~ms}$ discharges $(5 \mathrm{~Hz}$ repetition rate, $50 \%$ duty cycle) with and without a DL in steady-state plasmas at the same source parameters.

Shown in Fig. 11(a) is a measurement of the complete IVDF $5 \mathrm{~ms}$ into a pulsed discharge for an antenna frequency of $9.0 \mathrm{MHz}$, a source magnetic field of $1000 \mathrm{G}$, an expansion chamber magnetic field of $17 \mathrm{G}$, and a neutral pressure of 0.98 mTorr. For this set of parameters, the mirror ratio (upstream to downstream) is 60. The IVDF measurement includes 80 distinct laser frequencies and was averaged over 200 plasma pulses with a lock-in amplifier integration time of $1 \mathrm{~ms}$ (yielding an effective time resolution of a few milliseconds). Shown in Fig. 11(b) is the average of 200 measurements of the time-resolved floating potential fluctuation amplitude measured in the plasma source, approximately 100 $\mathrm{cm}$ upstream of the DL. Note the appearance of a welldefined, coherent oscillation after the large peak in fluctuation amplitude at $21.7 \mathrm{~ms}$ into the pulsed discharge. The initial wave amplitude is quite large and the envelope of the fluctuations decays exponentially after the initial large peak. The fact that this fluctuation waveform survives averaging over 200 pulses demonstrates the highly reproducible nature of these pulsed helicon discharges. Just before the large spike in fluctuation amplitude, at $21.5 \mathrm{~ms}$, the ion beam completely vanishes (Fig. 12). For this large expansion ratio case, the initial ion beam velocity, when the beam first appears in the measured IVDF, was $8.7 \mathrm{~km} / \mathrm{s}$ and the background ion population to beam density ratio is roughly $1: 1$. The time difference between the collapse of the DL (termination of the ion beam) and the peak in fluctuation amplitude, $\sim 0.2 \mathrm{~ms}$, is consistent with the $0.14 \mathrm{~ms}$ required for a wave to propagate from the DL location to the fluctuation measurement location at the local sound speed.

For the same source magnetic field, the same neutral 


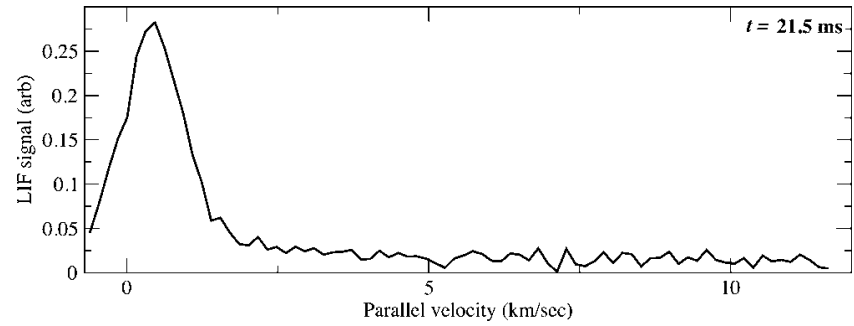

FIG. 12. For an antenna frequency of $9.0 \mathrm{MHz}$, the downstream IVDF $21.5 \mathrm{~ms}$ [0.2 ms prior to the spike in fluctuation amplitude in Fig. 11(b)] after the start of the $100 \mathrm{~ms}$ long pulsed discharge. The background population is evident, but the ion beam has vanished.

pressure, but for a larger expansion chamber magnetic field of $33 \mathrm{G}$ (which previous experiments demonstrated lead to reduced ion beam velocity, i.e., a weaker DL, because the mirror ratio of 30 is smaller than the previous case ${ }^{47}$ ), the ion beam persists throughout the discharge. The IVDF $25.5 \mathrm{~ms}$ into the pulsed discharge is shown in Fig. 13(a) and a clear ion beam is still discernible. Note that for this case, the background ion population to ion beam density ratio is greater than 2:1 and the initial ion beam velocity was $7.3 \mathrm{~km} / \mathrm{s}$. The floating potential fluctuation amplitude versus time for these plasma conditions is shown in Fig. 13(b). By $21.7 \mathrm{~ms}$ into the discharge, the time at which the large spike in fluctuation amplitude appeared in the previous case, the plasma potential fluctuation amplitude is nearly zero. As shown in the composite image in Fig. 14(a), for the larger mirror ratio, the initial beam population is actually larger than the background ion population at this measurement location. As the beam decays, the background ion population becomes much larger than the beam population. The floating potential fluctuation amplitude versus time is shown beneath the time-resolved IVDF in Fig. 14(a) and there is a clear anti-correlation between the fluctuation amplitude and the beam amplitude. When the fluctuation amplitude peaks, the beam amplitude is a minimum; leading to a "ripple" in both
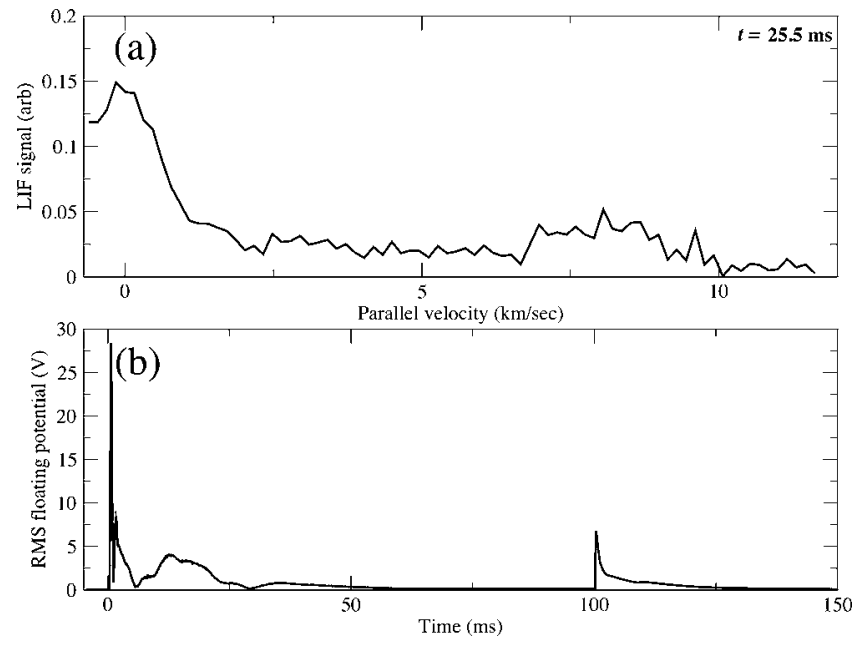

FIG. 13. For an antenna frequency of $12.0 \mathrm{MHz}$. (a) The downstream IVDF $25.5 \mathrm{~ms}$ after the start of the $100 \mathrm{~ms}$ long pulsed discharge. Both the background population (left) and ion beam (right) are evident. (b) Time series of the fluctuating floating potential from a single tip of the multitip probe averaged over 200 pulses.

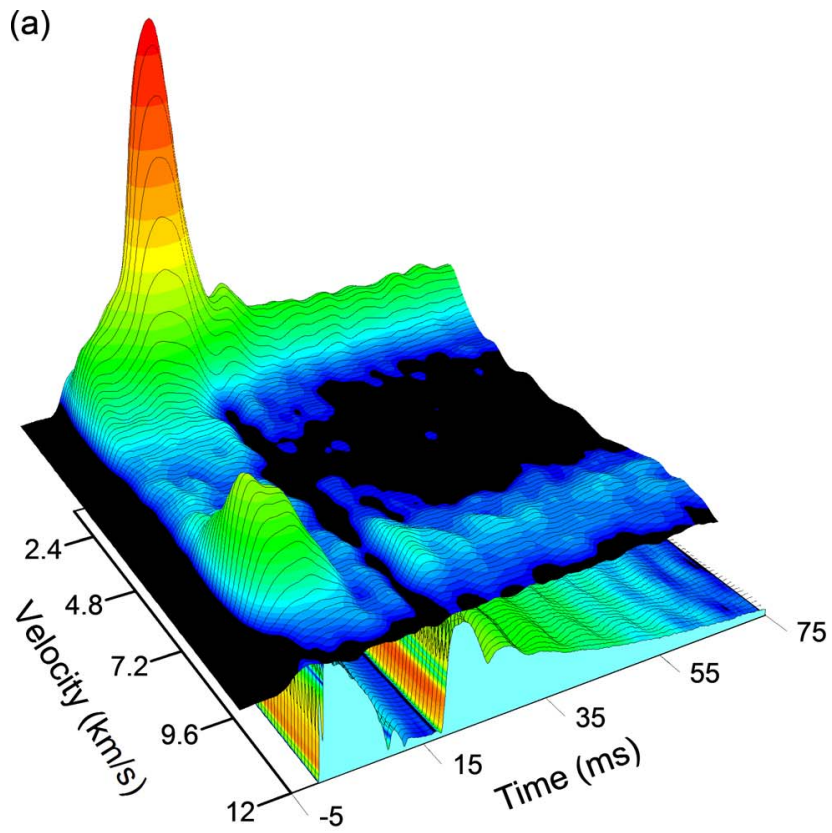

(b)

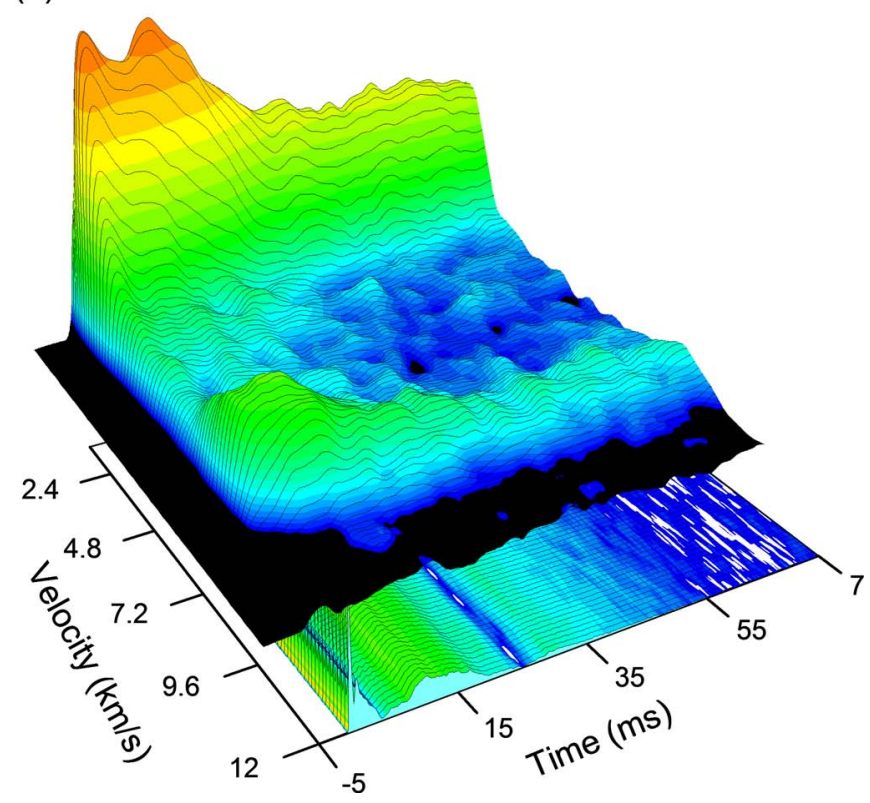

FIG. 14. (Color online) (a) For a mirror ratio of 60 (corresponding to Figs. 11 and 12), the upper surface plot is the IVDF vs time measured downstream of the DL. The ion beam appears at the start of the plasma pulse at a velocity of $\sim 8.7 \mathrm{~km} / \mathrm{s}$ and is larger than the background population. The lower surface plot is the floating potential vs time measured upstream of the DL. (b) The same measurements as in part (a) for a mirror ratio of 30 (corresponding to Fig. 13).

the IVDF and wave amplitude versus time. Shown in Fig. 14(b) are the same measurements for the smaller mirror ratio case. For the smaller mirror ratio, the relatively less dense (compared with the background ion population) and slower ion beam persists throughout the pulse and no instability is excited. The potential measurements shown in Fig. 14(b) are plotted on the same scale as used in Fig. 14(a) and it is clear that the fluctuation amplitude is much smaller for the smaller mirror ratio case. 


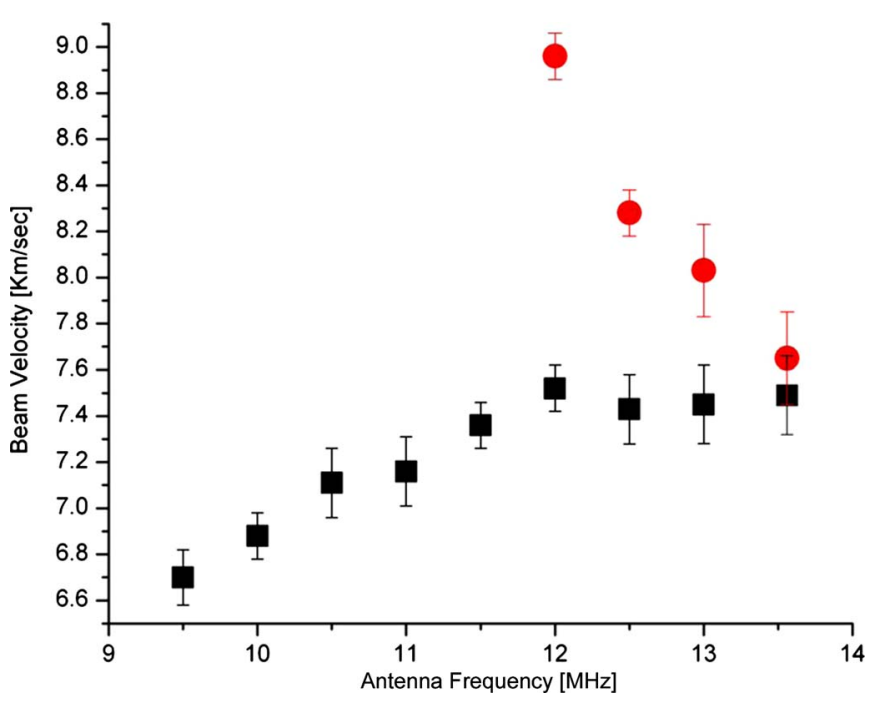

FIG. 15. (Color online) Upstream (squares) and downstream (circles) ion beam velocity vs antenna frequency. The velocities have been corrected for Zeeman shifts and the angle of the downstream LIF probe. The downstream ion beam vanishes for lower antenna frequencies. Reprinted with permission from Chakraborty Thakur et al., Phys. Rev. Lett. 102, 035004 (2009). Copyright @ 2009 American Physical Society.

\section{DISCUSSION}

We suggest the following interpretation of the observations reported here. At antenna frequencies below 11.5-12 $\mathrm{MHz}$, a strong DL forms and accelerates an ion beam to velocities greater than the sound speed. The accelerated ion and electron beam currents exceed a threshold for excitation of a current-driven instability and large electrostatic fluctuations develop; thereby destroying the strong potential gradient necessary for the DL and the DL collapses. The instabilities appear as large amplitude noise on Langmuir probe and RFEA measurements in steady-state discharges. The DL is stronger (the relative intensity of the ion beam is larger and the ion beam velocity is larger) at lower antenna frequencies because the coupling of rf energy into the plasma improves at lower antenna frequencies. Thus, it is at the higher antenna frequencies that the ion beam persists in both the pulsed and steady-state discharges.

Once the rf power coupling efficiency drops at higher antenna frequencies, a stable, but weaker, DL forms; the electrostatic noise is significantly reduced; and the ion beam appears downstream. Consistent with this hypothesis are the measurements of the upstream bulk ion speeds and the downstream ion beam velocities (obtained from the IVDFs of Figs. 4 and 5) shown in Fig. 15. The downstream ion beam velocity clearly increases with decreasing antenna frequency (the DL is getting stronger) until the beam abruptly vanishes downstream. The upstream beam velocity is relatively constant at the higher antenna frequencies and then begins to drop at the same threshold antenna frequency (11 $\mathrm{MHz}$ ) for which the downstream beam vanishes. In the timeresolved studies, two ion beam velocity and amplitude cases were obtained through two different divergent magnetic field mirror ratios. For the larger beam velocity and amplitude case, the instability grows while the DL collapses (the ion beam vanishes). For smaller beam velocity and relative am- plitude case, the ion beam persists throughout the discharge and no electrostatic fluctuations are observed; consistent with the higher beam velocity results obtained in the steady-state discharge experiments.

Because the growth of the instability disrupts the DL, these measurements provide a unique means of experimentally studying the physics related to the formation of a current-free DL in expanding plasmas. These experiments are not the first to indicate the presence of low frequency instabilities associated with current-free DLs (Ref. 45) or with DLs created in divergent magnetic fields. ${ }^{44}$ However, to the best of our knowledge, complete collapse of a DL correlated with the appearance of intense electrostatic instabilities has not been reported previously in a laboratory experiment. These results suggest that creation of strong DLs in expanding plasmas for plasma propulsion ${ }^{48,49}$ may be self-limited through instability growth and also demonstrate a mechanism for the collapse of naturally occurring DLs.

\section{ACKNOWLEDGMENTS}

This work was supported by NSF Award No. PHY-0611571. S.S. was supported by an Oak Ridge Institute for Science Education fellowship and J.C.J. was supported by a WV Space Grant graduate fellowship.

${ }^{1}$ N. Hershkowitz, IEEE Trans. Plasma Sci. 22, 11 (1994).

${ }^{2}$ H. Inuzuka, Y. Torri, M. Nagatsu, and T. Tsukishima, Phys. Fluids 28, 703 (1985).

${ }^{3}$ Y. Takeda and K. Yamgiwa, Phys. Rev. Lett. 55, 711 (1985).

${ }^{4}$ N. Sato, R. Hatakeyama, S. Iizuka, T. Mieno, K. Saeki, J. J. Rasmusten, and P. Michelsen, Phys. Rev. Lett. 46, 1330 (1981).

${ }^{5}$ S. Torvén, J. Phys. D 15, 1943 (1982).

${ }^{6}$ C. Chan, N. Hershkowitz, and T. Intrator, Phys. Rev. Lett. 52, 1782 (1984).

${ }^{7}$ N. Hershkowitz, Space Sci. Rev. 41, 351 (1985).

${ }^{8}$ R. L. Stenzel, M. Ooyama, and Y. Nakamura, Phys. Rev. Lett. 45, 1498 (1980).

${ }^{9}$ B. H. Quon and A. Y. Wong, Phys. Rev. Lett. 37, 1393 (1976).

${ }^{10}$ P. Coakley, N. Hershkowitz, R. Hubbard, and G. Joyce, Phys. Rev. Lett. 40, 230 (1978).

${ }^{11}$ N. Hershkowitz, G. L. Payne, C. Chan, and J. DeKock, Plasma Phys. 23, 903 (1981)

${ }^{12}$ P. Coakley and N. Hershkowitz, Phys. Fluids 22, 1171 (1979).

${ }^{13}$ P. Coakley and N. Hershkowitz, Phys. Lett. 83A, 131 (1981).

${ }^{14}$ G. Hairapetian and R. L. Stenzel, Phys. Rev. Lett. 61, 1607 (1988).

${ }^{15}$ L. P. Block, Astrophys. Space Sci. 55, 59 (1978).

${ }^{16}$ I. B. Bernstein, F. M. Greene, and M. D. Kruskal, Phys. Rev. 108, 546 (1957).

${ }^{17}$ C. Charles, A. W. Degeling, T. E. Sheridan, J. H. Harris, M. A. Lieberman, and R. W. Boswell, Phys. Plasmas 7, 5232 (2000).

${ }^{18}$ Z. Harvey, S. Chakraborty-Thakur, A. Hansen, R. A. Hardin, W. S. Przybysz, and E. E. Scime, Rev. Sci. Instrum. 79, 10F314 (2008).

${ }^{19}$ X. Sun, A. M. Keesee, C. Biloiu, E. E. Scime, A. Meige, C. Charles, and R. Boswell, Phys. Rev. Lett. 95, 025004 (2005).

${ }^{20}$ X. Sun, S. Cohen, and E. Scime, Phys. Rev. Lett. 93, 235002 (2004).

${ }^{21}$ C. Charles, Plasma Sources Sci. Technol. 16, R1 (2007).

${ }^{22}$ H. S. Byhring, C. Charles, A. Fredriksen, and R. W. Boswell, Phys. Plasmas 15, 102113 (2008).

${ }^{23}$ A. Keesee, E. E. Scime, C. Charles, A. Meige, and R. W. Boswell, Phys. Plasmas 12, 093502 (2005).

${ }^{24}$ X. Sun, S. Cohen, and E. E. Scime, Phys. Plasmas 12, 103509 (2005).

${ }^{25}$ N. Plihon, C. S. Corr, and P. Chabert, Appl. Phys. Lett. 86, 091501 (2005).

${ }^{26}$ C. Charles, R. W. Boswell, and R. Hawkins, Phys. Rev. Lett. 103, 095001 (2009).

${ }^{27}$ C. Charles and R. W. Boswell, Appl. Phys. Lett. 82, 1356 (2003).

${ }^{28}$ C. Charles, Appl. Phys. Lett. 84, 332 (2004). 
${ }^{29}$ C. Charles and R. W. Boswell, Phys. Plasmas 11, 3808 (2004).

${ }^{30}$ S. A. Cohen, N. S. Siefert, S. Stange, R. F. Boivin, E. E. Scime, and F. M. Levinton, Phys. Plasmas 10, 2593 (2003).

${ }^{31}$ M. A. Lieberman and C. Charles, Phys. Rev. Lett. 97, 045003 (2006); M.

A. Lieberman, C. Charles, and R. W. Boswell, J. Phys. D 39, 3294 (2006).

${ }^{32}$ S. Chakraborty Thakur, Z. Harvey, I. A. Biloiu, A. Hansen, R. A. Hardin,

W. S. Przybysz, and E. E. Scime, Phys. Rev. Lett. 102, 035004 (2009).

${ }^{33}$ E. Scime, P. Keiter, M. W. Zintl, M. M. Balkey, J. L. Kline, and M. E. Koepke, Plasma Sources Sci. Technol. 7, 186 (1998).

${ }^{34}$ I. A. Biloiu, E. Scime, and C. Biloiu, Plasma Sources Sci. Technol. 18, 025012 (2009).

${ }^{35}$ A. M. Keesee, R. Boivin, and E. Scime, Rev. Sci. Instrum. 75, 4091 (2004).

${ }^{36}$ E. Scime, C. Biloiu, C. Compton, F. Doss, J. Heard, E. Choueri, and S. Spektor, Rev. Sci. Instrum. 76, 026107 (2005).

${ }^{37}$ I. A. Biloiu and E. E. Scime, Appl. Phys. Lett. 95, 051504 (2009).

${ }^{38}$ C. Biloiu, E. E. Scime, and X. Sun, Rev. Sci. Instrum. 75, 4296 (2004).

${ }^{39}$ I. A. Biloiu, E. E. Scime, C. Biloiu, and S. Cohen, Proceedings of the
XXVIII International Conference on the Physics of Ionized Gasses, Prague (Institute of Physics, Bristol, 2007), p. 1537.

${ }^{40}$ M. Balkey, R. Boivin, P. A. Keiter, J. L. Kline, and E. Scime, Plasma Sources Sci. Technol. 10, 284 (2001).

${ }^{41}$ S. Iizuka, K. Saeki, N. Sata, and Y. Hatta, Phys. Rev. Lett. 43, 1404 (1979).

${ }^{42}$ C. Chan, N. Hershkowitz, and T. Intrator, Phys. Rev. Lett. 57, 3050 (1986).

${ }^{43}$ T. Sato and H. Okuda, Phys. Rev. Lett. 44, 740 (1980).

${ }^{44}$ M. J. Alport, S. L. Cartier, and R. L. Merlino, J. Geophys. Res. 91, 1599, doi:10.1029/JA091iA02p01599 (1986).

${ }^{45}$ A. Aanesland, C. Charles, M. A. Lieberman, and R. W. Boswell, Phys. Rev. Lett. 97, 075003 (2006).

${ }^{46}$ S. P. Gary and N. Omidi, J. Plasma Phys. 37, 45 (1987).

${ }^{47}$ C. Biloiu, X. Sun, E. Choueri, C. Compton, F. Doss, J. Heard, E. E. Scime, R. Specktor, and D. Ventura, Plasma Sources Sci. Technol. 14, 766 (2005).

${ }^{48}$ F. R. Chang-Diaz, Sci. Am. 283, 90 (2000).

${ }^{49}$ C. Charles, J. Phys. D 42, 163001 (2009). 